\title{
Influence d'un certain nombre de facteurs de croissance et de différents traitements thermiques du lactosérum sur la fermentation alcoolique du lactose par saccharomyces lactis
}

\author{
par
}

A. JABARIT

En raison de sa valeur biologique, le lactosérum est susceptible d'un emploi dans les industries alimentaires, dont en diététique, et même en thérapeutique. Sa valorisation doit encore s'étendre à la préparation d'aliments du bétail autant qu'à la fabrication de boissons rafraîchissantes.

Il nous a paru ainsi utile d'étudier la fermentation alcoolique du lactose en recherchant, d'une part, les facteurs de stimulation ou d'inhibition de ce processus, liés notamment à la présence de sels minéraux et d'acides aminés, et, d'autre part, l'influence exercée par les traitements thermiques.

La complexité du produit naturel, ici considéré, nous a incité à opérer en milieu plus simple afin de pouvoir mieux apprécier les actions éventuelles sur le lactose et sa fermentation, celle-ci étant réalisée par Saccharomyces lactis. Nous présentons ci-après, de manière abrégée, les données et les résultats de notre étude.

\section{PROTOCOLE EXPERIMENTAL}

- Préparation et stérilisation du milieu synthétique.

- Préparation d'une solution aqueuse avec la ou les substances pures à étudier. déterminé.

- Chauffage de cette solution selon un graphique thermique

- Mélange aseptique des solutions (milieu synthétique + solution aqueuse).

- Ensemencement à 1 p. 100 avec une culture du microbe réactif. 
- Détermination de la vitesse de croissance du microbe réactif par dosage régulier du lactose et de l'alcool présent dans le milieu, et contrôle systématique (au microscope, et par ensemencements en boîtes de Pétri) de l'état bactériologique du milieu au cours de l'expérience.

Notre but a été d'étudier l'influence du chauffage en appliquant différents types de traitement thermique sur les composants lactés.

Deux aspects de la question nous semblent importants :

- Influence du chauffage sur chaque composant du lactosérum pris séparément.

- Interaction entre les divers composants chimiques au cours du chauffage.

Le moyen pratique choisi a été de déterminer la vitesse de croissance de notre microbe réactif, Saccharomyces lactis, dans les différents essais. Or cette levure ne fermente que le lactose.

Deux idées principales nous ont guidé pour mettre au point notre protocole d'expérimentation :

- Séparer les essais de manière à ne faire varier qu'un seul facteur à la fois (chauffage ou composition du milieu). entre eux.

- Avoir la possibilité de comparer tous les résultats obtenus

Par conséquent, notre plan de recherche a été schématiquement celui-ci :

- Influence du chauffage sur des solutions de lactose chauffées, seules.

- Influence du chauffage sur des solutions de deux constituants du lactosérum chauffés ensemble : lactose plus sels minéraux, acides aminés, protides, etc.

- Influence du chauffage sur des solutions de lactose chauffées en présence de plus de deux constituants en mélange.

\section{METHODES UTILISEES}

Nous avons choisi le dosage à l'aide d'un micro-organisme.

Le dosage microbiologique est employé pour doser les vitamines et les acides aminés, mais on peut l'utiliser aussi pour la détermination d'une activité vitaminique totale $[1,10]$ selon le but poursuivi.

La technique de détection des facteurs de croissance de chaque micro-organisme est elle-même variable. William [11] se basait sur la numération des cellules et Bachmann [3] mesurait le gaz carbonique dégagé par les cultures pour déterminer la vitesse de croissance des cellules. Auclair et Portmann [2] ont employé des ferments 


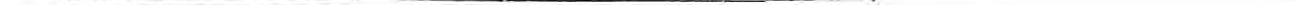


lactiques pour détecter les facteurs de croissance que l'on aurait détruits par le chauffage du lait [6-8], Keilling, Camus et Jabot [7], Claveau [4] ont utilisé Saccharomyces lactis pour étudier le vieillissement des produits laitiers.

\section{MODE OPERATOIRE}

Nous avons étudié les problèmes suivants :

- Choix du microbe-réactif.

- Choix du milieu de culture.

- Choix du test chimique et microbiologique.

Choix du microbe-réactif : L'agent de fermentation est Saccharomyces lactis. Il est conservé par repiquage sur un milieu de lactosérum gélosé à $\mathrm{pH}=7$.

L'ensemencement définitif est réalisé à partir d'une culture pure de cette levure cultivée sur lactosérum ayant un $\mathrm{pH}$ de 3,5 et âgée de $24 \mathrm{~h}$. La température d'incubation est de $25^{\circ} \mathrm{C}$ et le temps d'incubation varie entre 120 à 216 h suivant les conditions de chauffage et les substances introduites dans le milieu initial.

Choix du milieu de culture: Le milieu synthétique employé est un milieu simple désigné sous le nom « milieu tomate-levure » dont voici la composition :

Jus de tomates $\ldots . .200 \mathrm{ml} \quad(500 \mathrm{~g}$ de tomates $+500 \mathrm{ml}$ - Jus de tomates ....... . $200 \mathrm{ml}\left\{\begin{array}{l}\text { d'eau distillée, bouillie, fil- } \\ \text { trée et centrifugée). }\end{array}\right.$

- Eau de levure........ $800 \quad\left\{\begin{array}{l}\text { langerie }+800 \mathrm{ml} \text { d'eau } \\ \text { distillée mélangée, agitée, }\end{array}\right.$ centrifugée).

- Phosphate bipotassique .. $1 \mathrm{~g} / \mathrm{l}$ ( on ajuste le $\mathrm{pH}$ de cette

- Sulfate de magnésium ... .. $0,5 \mathrm{~g} / 1$ lolution à 5,7 avant stéri-

- Phosphate monocalcique .. $3 \mathrm{~g} / 1$ lisation.

On stérilise à $118^{\circ} \mathrm{C}$ pendant $25 \mathrm{mn}$, le $\mathrm{pH}$ final est de 5,4.

Ce milieu de culture est ajouté à la solution à étudier. Dans tous les cas, des contrôles sur boîtes de Pétri ont été effectués pour vérifier qu'il n'y avait pas de contamination.

Choix du test chimique et microbiologique : Pour mesurer la vitesse de croissance de la levure, nous avons choisi les méthodes analytiques suivantes :

- Numération microbienne sur les milieux spécifiques (dénombrement microbien).

- Mesure d'une variation physico-chimique du milieu provoquée par le métabolisme de la levure (alcool, gaz, etc.). 


\section{IDENTIFICATION DU MICROBE-REACTIF}

- Origine : Il s'agit d'un Saccharomyces qui a été isolé de la surface d'un Camembert au début de sa maturation.

- Aspect microscopique : Cellules ovoïdes à protoplasme régulier et bourgeonnement - dimension : 3,5 à 5 microns.

- Caractères culturaux :

a) sur gélose nutritive : colonies circulaires donnant après vieillissement de grandes colonies à bords larges et minces avec des prolongements arborescents ;

b) sur gélatine nutritive : colonies géantes montrant au centre l'aspect d'un cratère aux bords relevés, entouré d'une large surface sensiblement circulaire, coupée par rayons ;

c) en gélatine nutritive en piqûre : développement le long de la piqûre ; la prolifération microbienne s'intensifiant régulièrement du fond vers la surface.

\section{IDENTIFICATION DE LA SOUCHE}

a) aspect des levures : cellules rondes, ovales, allongées, seules ou par couple, chaque asque ayant 1 à 4 ascospores ;

b) caractères biochimiques :

- sucres fermentés : glucose, galactose, saccharose et lactose complètement, et raffinose au $1 / 3$; lactose ;

- sucres assimilés : glucose, galactose, saccharose, maltose et

- utilisation de l'éthanol comme source de carbone comparé à la croissance sur milieu de Raulin.

Par les caractères que nous avons constatés, nous pouvons identifier notre souche à Saccharomyces lactis beta de Dombrowski cu Saccharomyces fragilis de Jorgensen. Ces deux formes paraissent être identiques, d'après Guillermond [5], Lodder et Kreger Von Rij [9].

\section{EXPOSE DES RECHERCHES EFFECTUEES}

- Obtention d'une solution de lactose à 5 p. 100 .

- Solution de lactose + sels minéraux (Phosphate monocalcique, Phosphate bipotassique, sulfate de magnésium et de calcium).

- Solution de lactose + acides aminés (Tyrosine, Histidine, Proline, Méthionine, Tryptophane et acide glutamique).

- Solutions composées : 
1) chauffage des solutions de lactose en présence des acides aminés et des sels minéraux qui accélèrent la fermentation ;

2) chauffage de lactosérum, établissement des courbes de fermentation des solutions de lactosérum chauffées à $120^{\circ} \mathrm{C}$ pendant $30 \mathrm{mn}$.

\section{VUE GENERALE SUR LES RESULTATS OBTENUS}

Pour rendre plus rapidement saisissable les résultats que nous avons obtenus, nous avons rassemblé dans deux tableaux, l'influence des conditions de chauffage d'une part, et les actions des diverses substances (facteurs de croissance) que l'on ajoute aux solutions de lactose, d'autre part, sur le temps total de fermentation.

En nous plaçant uniquement du point de vue des influences des composants additionnés à la solution de lactose, nous pouvons constater des effets de ralentissement et des effets d'accélération. Il est donc possible de réaliser un milieu synthétique qui se comporte comme le lactosérum naturel.

Après de nombreux tâtonnements, nous avons pu mettre au point un tel milieu synthétique.

\section{CONCLUSION GENERALE}

Nous sommes arrivés à des résultats reproductibles, indiquant des vitesses de fermentation différentes en fonction des facteurs de croissances du milieu, en révélant ainsi des variations qui, autrement, risqueraient de passer inaperçues.

Nous avons mis en évidence les points suivants :

a) la chaleur exerce peu d'effet sur le comportement du glucide en solution pure, ou en solutions enrichies soit de composés minéraux, soit d'acides aminés. La cinétique et la durée de la fermentation sont un peu modifiées lorsque les températures de chauffage préalables ont atteint les valeurs de celles dites de pasteurisation ; aux températures supérieures, les vitesses de fermentation augmentent à nouveau pour devenir semblables à celles du témoin non chauffé ;

b) le chauffage du lactose en présence de sels minéraux nous a montré que certains anions ou cations agissaient spécifiquement sur le sucre. Il nous est apparu, en particulier, que le calcium avait une action nuisible, action qui est compensée par la présence d'autres sels : sulfate de magnésium, phosphate bipotassique ;

c) la présence d'acides aminés au cours du chauffage du lactose n'a que peu d'influence sur la marche de la fermentation qui est ensuite produite par la levure. 
Nous avons classé quantitativement les acides aminés en trois groupes :

a) la Tyrosine et l'acide Glutamique agissent dans un sens d'accélération ;

b) la Méthionine et le Tryptophane ont un faible effet d'accélération ;

c) la Proline et l'Histidine jouent un rôle inhibiteur ;

d) enfin, en combinant diverses substances, nous avons obtenu un milieu synthétique qui se comporte, du point de vue de la vitesse de fermentation par la levure, comme le lactosérum naturel.

\section{$R$ é s u $\mathrm{m}$ é}

Le chauffage seul n'intervient que très peu, directement, sur le lactose du lactosérum, mais l'action des sels minéraux, ne serait-ce qu'à l'état d'impuretés, peut être beaucoup plus sensible, surtout s'il s'agit du calcium. Ainsi, cet élément peut jouer un rôle important lors du chauffage des solutions de lactose, en sucrerie, condenserie, confiserie, préparation d'aliments du bétail et de boissons rafraîchissantes, ou encore pour la diététique. Il s'ensuit que, dans ces industries, la qualité des eaux devrait être appréciée avec soin, ce qui pourrait, éventuellement, entraîner la mise en œuvre d'utiles mécanismes de correction quant à leur teneur en sels de calcium.

\section{S u m m a ry}

Directly, heat treatment very little influences lactose of whey, but the effect of mineral salts, even if in very small quantities, is liable to be more important, especially as regards calcium.

So this element can play an important part during the heating of lactose solutions in sugar factories, preparation of refreshing drinks, of cattle food. Hence follows that in these industries the quality of water should be tested carefully in order to bring forth the application of useful corrective techniques as to their calcium compounds.

Reçu pour publication en septembre 1970.

\section{Bibliographie essentielle}

[1] Adrian (J.) (1953). - Le dosage microbiologique des vitamines du groupe B. Ann. Nutr. et Alim., 13.

[2] Auclair et Portmann (1956). - Influence du chauffage du lait aux températures de pasteurisation et de stérilisation sur la croissance des bactéries lactiques. XIV Congrès Inter. sur le lait et ses dérivés, Rome, I, p. 17.

[3] Bachmann (1919). - Vitamin requirements of certains yeast. Biol. Chem., $39,235$. 
[4] Claveau (1953). - Influence de la nicotinamide sur une levure du lactose, application pratique à un procédé de dosage microbiologique de cette vitamine dans les produits alimentaires. Thèse doctorat de Lille.

[5] Guillermond (A.) (1912). - Les levures. 1 vol. collection encyclopédie scientifique, Doin éd., Paris.

[6] Jacouet (J.) (1961). - De l'importance et de l'intérêt actuels des études concernant le lait (galactologie). Mém. Acad. Sci. Arts et Belles Lettres de Caen.

[7] Keilling (J.), Camus (A.) et Jabot (P.) (1947). - Note sur un aspect du vieillissement des produits laitiers en poudre. Bull. Sté Scient. Hyg. Alim., 194.

[8] Kon (S. K.) (1958). - Influence of heat treatment and hight on the composition and quality of milk. Dairy Sci. Abstr., 20, 887.

[9] Lodder (J.) and KReger von RiJ (1952). - The yeasts, a taxonomic study. 1 vol., North Holland Publish. comp., Amsterdam.

[10] Pottenger (F.) (1946). - The effect of heat processed and metabolized vitamin $\mathrm{D}$ milk on the dentofacial structures experimental animals. Am. J. Orthod, and Oral Surgery, 32.

[11] Williams (J. R.) (1919). - The vitamin requierment of yeast : a simple biological test by vitamin. J. Biol. Chem.

\section{Remerciements}

Nous remercions M. le Pr Keilling notre Maître et notre guide pour la réalisation de ce travail.

Nos remerciements s'adressent également à M. le Pr Jacquet, professeur à la Faculté des sciences de Caen (Calvados), pour son concours scientifique. Nous tenons à remercier M. le Pr Thieulin pour les précieux conseils qu'il a bien voulu nous donner pour la rédaction de cet article. 